\title{
SELETIVIDADE DE MISTURAS DE HERBICIDAS E INSETICIDAS EM TANQUE APLICADAS EM HÍBRIDOS DE MILHO
}

\author{
CLEBER DANIEL DE GOES MACIEL ${ }^{1}$, ANDRÉ AUGUSTO PAZINATO DA SILVA ${ }^{1}$, \\ ENELISE OSCO HELVIG ${ }^{1}$, ANTÔNIO MENDES DE OLIVEIRA NETO ${ }^{2}$, NAIARA GUERRA ${ }^{3}$, \\ LUIZ CARLOS SOLA JÚNIOR ${ }^{4}$ e DÉCIO KARAM ${ }^{5}$
}

\author{
${ }^{1}$ Universidade Estadual do Centro-Oeste, 85040-080, Campus de CEDETEG, Guarapuava-PR, Brasil. \\ cmaciel@unicentro.br; andrepazinato0@gmail.com; eneosco@hotmail.com; \\ ${ }^{2}$ Instituto Federal Catarinense, 89163-356, Campus Rio Grande do Sul, Rio do Sul-SC, Brasil. \\ am.oliveiraneto@gmail.com \\ ${ }^{3}$ Universidade Federal de Santa Catarina, 89520-000, Campus Curitibanos, Curitibanos-SC, Brasil.naiara.guerra@hotmail.com \\ ${ }^{4}$ Cooperativa Casul,19.600-000,Rancharia-SP,Brasil.luiz@casul.com.br; \\ ${ }^{5}$ Embrapa Milho e Sorgo, 35701-970, Sete Lagoas-MG, Brasil.decio.karam@embrapa.br
}

Revista Brasileira de Milho e Sorgo, v.17, n.2, p. 287-302, 2018

\begin{abstract}
RESUMO - A associação de herbicidas e inseticidas é uma prática comum entre os agricultores, entretanto, muitas vezes pode provocar injúrias à cultura do milho. O trabalho teve como objetivo avaliar a seletividade de herbicidas em mistura em tanque com inseticidas de diferentes grupos químicos, no crescimento inicial de hibridos de milho. Dois experimentos foram conduzidos separadamente em estufa plástica com dois híbridos de milho (30F35 e 2B-710), utilizando como unidades experimentais vasos com duas plantas nos estádios fenológicos $\mathrm{V}_{3}$ e $\mathrm{V}_{6}$. $\mathrm{O}$ delineamento experimental foi o inteiramente casualizado, com cinco repetições. Foram avaliados 31 tratamentos, representados pela aplicação de seis herbicidas (atrazine, nicosulfuron, foramsulfuron + iodosulfuron, mesotrione, carfentrazone e 2,4-D, nas doses de 2000;37,5;36+2,4;144; 10 e $1000 \mathrm{~g} \mathrm{ha}^{-1}$, respectivamente) isolados e em mistura com quatro inseticidas (permethrin, methomyl, chlorpyrifos ethyl e novarulon, nas doses de 26,9; 64,5; 240 e $15 \mathrm{~g} \mathrm{ha}^{-1}$, respectivamente) e uma testemunha sem aplicação. Avaliou-se a intoxicação das plantas aos 7 e 14 dias após a aplicação (DAA), o teor de clorofila das folhas, número médio de folhas por planta, massa seca da parte aérea e das raízes aos 14 DAA. Os resultados foram variáveis em função do híbrido e estádio fenológico na aplicação, sendo as misturas menos prejudiciais ao milho quando aplicadas em $\mathrm{V}_{3}$. Os tratamentos com atrazine foram os que menos afetaram o crescimento inicial dos híbridos. As misturas nicosulfuron + chlorpyrifos ethyl, mesotrione + chlorpyrifos ethyl e 2,4-D + chlorpyrifos ethyl foram as que mais prejudicaram o híbrido 30F35. Por outro lado, a mistura carfentrazone + chlorpyrifos ethyl foi a que mais afetou o crescimento inicial do híbrido 2B-710.
\end{abstract}

Palavras-chave: fitointoxicação, antagonismo, estádio de desenvolvimento, Zea mays.

\section{SELECTIVITY OF HERBICIDES AND INSECTICIDES TANK MIXTURES APPLIED IN MAIZE HYBRIDS}

\begin{abstract}
The association of herbicides and insecticides despite often cause damage to the corn crop is a common practice among farmers. This study aimed to evaluate the initial selectivity of herbicides in tank mixture with insecticides from different chemical groups in maize. Two experiments were carried out in plastic greenhouse, using as experimental units pots with two maize hybrids $(30 \mathrm{~F} 35$ and $2 \mathrm{~B}-710)$ in $\mathrm{V}_{3}$ and $\mathrm{V}_{6}$ phenological stages. A completely randomized experimental design was used, with five replications. Thirty-one treatments were represented by applying six herbicides (atrazine, nicosulfuron, foramsulfuron+iodosulfuron, mesotrione, carfentrazone and 2.4D) isolated and in combination with the four insecticides (permethrin, methomyl, chlorpyrifos ethyl and novarulon) and a check without application. The phytointoxication was evaluated at 7 and 14 days after application (DAA), the chlorophyll content in leaves, total number of leaves, dry mass of the aerial part and roots at 14 DAA. The results were dependent of hybrid and corn stage in the application, and mixtures were less harmful when applied in $\mathrm{V}_{3}$ stage. The treatments with atrazine affected less the initial growth of the two hybrids. The mixtures of nicosulfuron+chlorpyrifos ethyl, mesotrione+chlorpyrifos ethyl and 2.4-D + chlorpyrifos ethyl were the most prejudicial to the hybrid 30F35. In addition, the carfentrazone + chlorpyrifos ethyl mixture was the most harmful to the initial growth of the hybrid $2 \mathrm{~B}-710$. Keywords: phytointoxication, antagonism, development stage, Zea mays.
\end{abstract}


A adição de inseticidas na calda de pulverização dos herbicidas pode modificar a capacidade da planta de tolerá-los e, consequentemente, interferir na seletividade e/ou controle das plantas daninhas (Rezende et al., 2012). Portanto, a seletividade dos herbicidas não é absoluta e pode resultar em prejuízos severos às culturas se aplicados em condições desfavoráveis ou de forma inadequada (Azania \& Azania, 2014).

Em algumas culturas anuais tais como milho, soja e algodão, o estádio de aplicação de herbicidas e inseticidas em pós-emergência pode ser simultâneo o que faz com que seja frequente entre os agricultores a prática de mistura desses defensivos agrícolas no tanque de pulverização, visando reduzir custos operacionais. Uma vez que não há defensivos que possuem espectro de ação capaz de controlar plantas daninhas e insetos concomitantemente, o uso de misturas em tanque tem sido realizado no Brasil e em outros países (Gazziero, 2015).

É importante ressaltar que a seletividade se caracteriza pelo resultado conjunto de diferentes mecanismos que protegem a cultura da intoxicação pelos herbicidas, com destaque para o metabolismo diferencial desses produtos entre plantas daninhas e cultivadas (Carvalho et al., 2009). Nesse sentido, Zanatta et al. (2007) relataram que interações sinergísticas de produtos podem reduzir a margem de segurança para as culturas, causando sintomas de fitotoxicidade, redução da altura, da biomassa seca da parte aérea e de raízes, e até mesmo causar a morte das plantas. Segundo os autores, muitos fatores afetam o grau de interação entre os herbicidas e inseticidas, com destaque para a forma de absorção e translocação, o método de aplicação, o estádio de desenvolvimento, tipo de formulação, características do solo e até mesmo a disponibilidade hídrica.
Na cultura do milho, os principais relatos de problemas da interação na mistura de herbicidas e inseticidas ocorrem com os organofosforados e metilcarbamatos (Steckel et al., 2015). López-Ovejero et al. (2003) mencionaram que a aplicação de misturas dos herbicidas [atrazine + metolachlor] + nicosuluron $\left([1000+1500]+20 \mathrm{~g} \mathrm{ha}^{-1}\right)$ e [atrazine + óleo vegetal] + nicosulfuron $\left([800+600]+20\right.$ a $\left.52 \mathrm{~g} \mathrm{ha}^{-1}\right)$ em milho deve ser realizada até o estádio fenológico $\mathrm{V}_{4}$ para evitar danos ao rendimento da cultura. Ao contrário de Silva et al. (2005) que relataram que a mistura em tanque de [atrazine + óleo] + nicosulfuron $([1200+$ $\left.900]+28 \mathrm{~g} \mathrm{ha}^{-1}\right)$ com o chlorpyrifos ethyl $\left(240 \mathrm{~g} \mathrm{ha}^{-1}\right)$ reduziu a massa seca das plantas do híbrido de milho P30F80 em aproximadamente 95, 80 e 46\%, em aplicações realizadas nos estádios fenológicos $\mathrm{V}_{2}, \mathrm{~V}_{4} \mathrm{e}$ $\mathrm{V}_{6}$, respectivamente.

Para Kreuz e Fonn-Pfister (1992) os inseticidas organofosforados e herbicidas inibidores da enzima acetolactato sintase (ALS), tais como o nicosulfuron, são degradados pelo mesmo sistema enzimático em milho, sendo que na presença do inseticida a taxa de metabolização do herbicida reduz, permitindo seu acúmulo em níveis que causam sintomas de intoxicação. Acredita-se que os organofosforados influenciam principalmente o metabolismo diferencial das plantas alterando a concentração de enzimas P450 (Yasour et al., 2009; Matzenbacher et al., 2015). Este complexo enzimático ocorre abundantemente nas plantas, sendo responsável pela metabolização e detoxificação de herbicidas (Nelson \& Werck-Reichhart, 2011; Dalazen et al., 2016).

$\mathrm{Na}$ literatura nacional ainda são poucos os relatos de interações antagônicas entre herbicidas como atrazine, nicosulfuron, foramsulfuron + iodosulfuron, mesotrione, carfentrazone, 2,4-D e inseticidas de diferentes grupos químicos na cultura do milho. As 
informações mais predominantes referem-se a intoxicações causadas por associações de nicosulfuron e chlorpyrifos ethyl, com sintomas de clorose, morte da gema apical e perfilhamento das plantas (Silva et al., 2005; Nicolai et al., 2006). Todavia, o uso conjunto de ingredientes ativos em mistura de tanque pode provocar efeitos adversos sobre as plantas daninhas e a cultura e, assim, tornam-se indispensáveis pesquisas sobre as prováveis interações entre herbicidas, indicando o uso mais adequado dessas associações.

Desta forma, o presente trabalho teve como objetivo avaliar a seletividade de herbicidas aplicados em mistura em tanque com inseticidas, no crescimento inicial de dois híbridos de milho nos estádios fenológicos $V_{3}$ e $V_{6}$.

\section{Material e Métodos}

Dois experimentos foram conduzidos separadamente em estufa plástica com os híbridos de milho 30F35 e 2B-710, utilizando como unidades experimentais vasos com $10 \mathrm{~kg}$ de solo e duas plantas de cada híbrido, nos estádios fenológicos $\mathrm{V}_{3}$ e $\mathrm{V}_{6}$.

O solo utilizado foi Latossolo Vermelho distroférrico, de textura arenosa. A análise físico-química, em amostras de 0 a $20 \mathrm{~cm}$ de profundidade, apresentou $\mathrm{pH}\left(\mathrm{CaCl}_{2}\right)$ de 5,$5 ; 17,0 \mathrm{mmol}_{\mathrm{c}} \mathrm{dm}^{-3}$ de $\mathrm{H}^{+}+\mathrm{Al}^{3+}$;

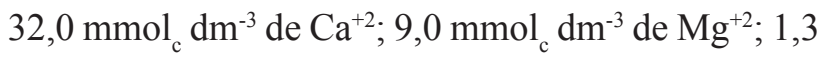
$\mathrm{mmol}_{\mathrm{c}} \mathrm{dm}^{-3}$ de K $\mathrm{K}^{+}$; 32,0 $\mathrm{mg} \mathrm{dm}^{-3}$ de P; 12,0 $\mathrm{g} \mathrm{dm}^{-3} \mathrm{de}$ MO; SB de 42,0 $\mathrm{mmol}_{\mathrm{c}} \mathrm{dm}^{-3}$; CTC de 59,0 $\mathrm{mmol}_{\mathrm{c}} \mathrm{dm}^{-3}$; V\% de $71,0 \%$, e 80,$3 ; 12,2$ e $7,5 \%$ de areia, argila e silte, respectivamente.

A estufa plástica modelo arcopampeana encontra-se disposta no sentido norte-sul no campus urbano da Escola Superior de Agronomia de Paraguaçu Paulista/SP (ESAPP), na região do médio Vale do Para-

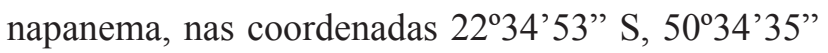

$\mathrm{O}$ e a $506 \mathrm{~m}$ de altitude. Essa estrutura possui $250 \mathrm{~m}^{2}$ ( $25 \times 10 \mathrm{~m})$, pé direito de $1,9 \mathrm{~m}$ e altura central de 3,3 $\mathrm{m}$, cobertura e cortinais lateriais móveis para ventilação, de polietileno de baixa densidade (PEBD) de 100 micras de espessura e aditivo anti-UV, com portas nas extremidades.

Os experimentos foram dispostos separadamente em delineamento inteiramente casualizado, com 31 tratamentos e 5 repetições para cada hírido de milho (30F35 e 2B-710). No entanto, para obtenção dos dois estádios fenológicos de cada híbrido na mesma unidade experimental, estes foram semeados com intervalo de dez dias, e após a emergência foi realizado desbaste, deixando apenas uma planta por unidade experimental nos estádios de três folhas $\left(\mathrm{V}_{3}\right)$ e seis folhas $\left(\mathrm{V}_{6}\right)$ totalmente expandidas.

Os tratamentos para cada experimento foram constituidos pela associação entre seis herbicidas (atrazine; nicosulfuron; foramsulfuron + iodosulfuron; mesotrione; carfentrazone e 2,4-D, nas doses de $2000 ; 37,5 ; 36+2,4 ; 144 ; 10$ e $1000 \mathrm{~g} \mathrm{ha}^{-1}$, respectivamente) e quatro inseticidas de diferentes grupos químicos (piretroide - permethrin; carbamato - methomyl; organofosforado - chlorpyrifos ethyl e inibidor de quitina - novarulon 26,9; 64,5; 240 e $15 \mathrm{~g} \mathrm{ha}^{-1}$, respectivamente) e uma testemunha sem aplicação. Em função da padronização dos tratamentos não foi acrescido nenhum tipo de adjuvante nas caldas de pulveriação.

A adubação de semeadura foi realizada por unidade experimental utilizando como referência 450 $\mathrm{kg} \mathrm{ha}^{-1}$ da fórmula 4-14-8 (N-P-K). Aos sete dias após a aplicação dos tratamentos realizou-se, de forma semelhante, a adubação de cobertura considerando 150 $\mathrm{kg} \mathrm{N} \mathrm{ha-1}$ na forma de ureia. O teor de água no solo dos vasos foi mantido próximo à capacidade de campo, por meio de duas irrigações diárias. 
As aplicações foram realizadas com pulverizador costal pressurizado a $\mathrm{CO}_{2}$ à pressão constante de $207 \mathrm{kPa}$, equipado com quatro pontas de jato plano DG11002 VS, em taxa de aplicação equivalente a $200 \mathrm{~L} \mathrm{ha}^{-1}$. As condições climáticas no momento da aplicação foram em média de: temperatura do ar de $26^{\circ} \mathrm{C}$, umidade relativa de $68 \%$, ventos de aproximadamente $1,5 \mathrm{~km} \mathrm{~h}^{-1}$, solo úmido e céu claro com ausência de nuvens.

Foram avaliados os sintomas de intoxicação visual dos híbridos, em porcentagem, em que $0 \%$ corresponde à ausência de injúrias e 100\% à morte das plantas aos 7 e 14 dias após a aplicação (DAA) (Velini et al., 1995). Avaliou-se também o teor de clorofila das penúltimas folhas expandidas aos 14 DAA, utilizando-se clorofilômetro portátil Modelo Minolta $^{\circledR}$ (índice SPAD), a altura das plantas do solo até o máximo de curvatura das folhas, o número total de folhas, assim como a massa seca total da parte aérea e das raízes.

Os dados obtidos foram submetidos à análise de variância pelo teste $\mathrm{F}$ e comparadas pelo teste de agrupamento de médias de Scott-Knott, a 5\% de probabilidade $(\mathrm{p} \leq 0,05)$.

\section{Resultados e Discussão \\ Descrição dos sintomas visuais de intoxicação}

A descrição dos sintomas visuais de intoxicação foi baseada no herbicida utilizado na mistura em tanque. Neste tópico não se tratou da intensidade dos sintomas, da sensibilidade diferencial entre os híbridos e do estádio de aplicação, uma vez que foram descritos nos resultados de seus respectivos experimentos. Os sintomas podem ser conferidos na Figura 1a e 1b, para o híbrido 30F35, e Figura 1c e 1d, para o híbrido 2B-710.
Para atrazine, sintomas de intoxicação foram visualizados apenas na mistura de atrazine + novarulon, caracterizados por leve clorose nas folhas adjacentes ao cartucho (Figura $1 \mathrm{a}$ e b). Esse sintoma apresentou baixa intensidade e nos demais tratamentos foi imperceptível. A seletividade do herbicida atrazine ao milho está associada à elevada capacidade desta espécie em converter atrazine em metabólitos derivados, principalmente hydroxyatrazine. Em milho, a rápida hidrólise deve-se às elevadas concentrações de bezoxazinona em suas células. Os produtos da metabolização de atrazine são rapidamente acumulados no vacúolo e perdem a mobilidade na planta (Raveton et al., 1997; Cherifi et al., 2001).

Inicialmente, os herbicidas nicosulfuron e foramsulfuron + iodosulfuron aplicados isolados ou associados aos inseticidas ocasionaram manchas de coloração amarelada na base das folhas da pare aérea ("cartucho") dos dois híbridos. Posteriormente, as folhas se dispuseram em posição ereta, com torção do limbo foliar em movimento de rotação no sentido horário (Figura $1 \mathrm{a}, \mathrm{b}, \mathrm{c}$ e d). Sintomas semelhantes foram relatados por Trezzi et al. (2005), para a aplicação de foramsulfuron + iodosulfuron no híbrido AG 3010, assim como por Cavalieri et al. (2012), que identificaram sintomas de clorose e alterações na morfologia foliar de híbridos de milho tratados com nicosulfuron.

A aplicação de mesotrione causou intoxicação visual descrita como leve clorose nas folhas dos híbridos, com evolução para branqueamento intenso somente para a mistura com chlorpyrifos ethyl (Figura $1 \mathrm{a}, \mathrm{b}, \mathrm{c}$ e d). Esta sintomatologia já era esperada, uma vez que o branqueamento é o principal sintoma visualizado após a aplicação de herbicidas inibidores da biossíntese de carotenoides, como o mesotrione (Steckel et al., 2015).

Para o agrupamento de tratamentos com carfentrazone, os sintomas de intoxicação foram seme- 


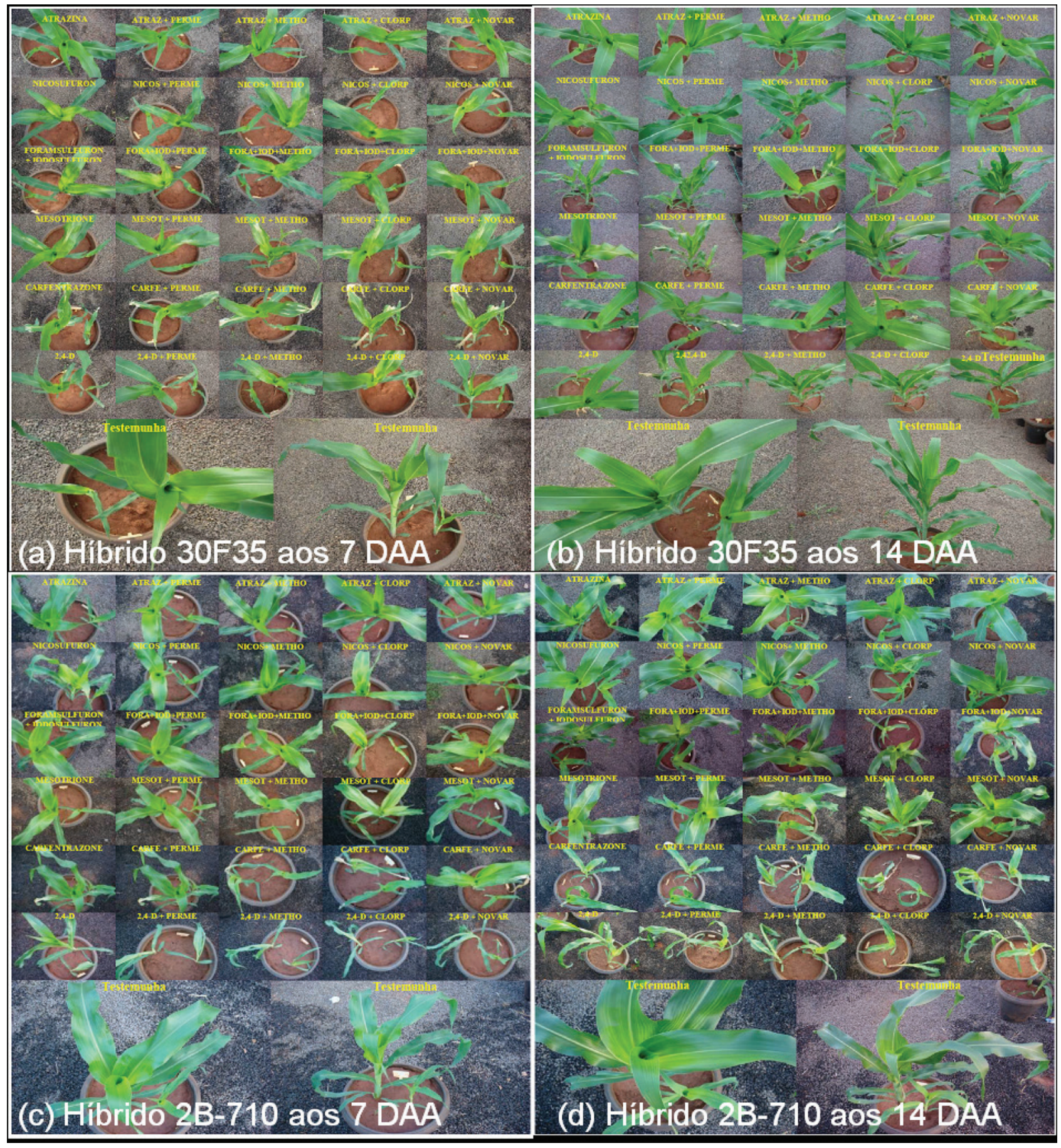

Figura 1. Ilustração dos sintomas de intoxicação em milho híbrido $30 \mathrm{~F} 35$ (a e b) e 2B-710 (c e d) aos 7 e 14 DAA (dias após aplicação), causados pelas associações dos herbicidas atrazine (ATRAZ), nicosulfuron (NICOS), foramsulfuron + iodosulfuron (FORA+IOD), mesotrione (MESOT), carfentrazone (CARFE) e 2,4D com os inseticidas permethrin (PREME), methomyl (METHO), chlorpyrifos ethyl (CLORP) e novarulon (NOVAR), aplicadas nos estádios fenológicos $\mathrm{V}_{3}$ e $\mathrm{V}_{6}$. 
lhantes e caracterizados por necrose e manchas pontuais, principalmente no centro e bordos das folhas mais velhas (Figuras 1, 2, 3 e 4). Estes sintomas estão diretamente relacionados ao modo como este herbicida atua na planta, já que a inibição da enzima protoporfirinogênio oxidase (PROTOX) leva ao acúmulo e transporte de protoforfirinogênio IX do cloroplasto para o citoplasma. Neste local, reações de oxidação formam protoporfirina IX que, na presença de luz e oxigênio, produz elevadas quantidades de oxigênio reativo, resultando na peroxidação dos lipídios e necrose de tecidos. Esses sintomas ficaram restritos às folhas mais velhas, pois o carfentrazone apresenta ação de contato (Vidal et al., 2014).

A aplicação de 2,4-D provocou injúrias descritas como manchas necróticas nas bordas e ponta das folhas mais velhas, até a redução do porte e inclinação parcial e/ou tombamento das plantas por completo (Figura 1 a, b, c e d). O sintoma de inclinação e/ou tombamento, também conhecido popularmente como "chicote", é resultante do crescimento irregular das células da base das plantas. Os primeiros sintomas observados do herbicida 2,4-D em milho são o encarquilhamento das folhas, o encurvamento da folha sobre a face inferior, as hastes curvadas para o solo (pescoço de ganso) e os órgãos mal constituídos (Karam \& Oliveira, 2007). Vieira Júnior et al. (2015) mencionaram que a aplicação da mistura em tanque de glyphosate $+2,4-\mathrm{D}$ resultou em sintomas de curvatura e aumento do diâmetro do colmo, além de redução do número de fileiras de grãos na espiga no híbrido P30F53HX RR.

\section{Experimento 1: híbrido 30F35}

A aplicação dos tratamentos no estádio $\mathrm{V}_{3}$ prejudicou o crescimento inicial do híbrido 30F35, já que as variáveis intoxicação visual, teor de clorofila, número de folhas por planta, massa seca da parte aérea e massa seca das raízes foram afetadas (Tabela 1). Apenas a altura de plantas não foi afetada pela aplicação dos tratamentos em $\mathrm{V}_{3}$.

A intensidade dos sintomas de intoxicação (\%) variou conforme o tratamento aplicado, aos 7 e 14 DAA, e as médias foram agrupadas em seis e sete grupos distintos pelo teste de Scott-Knott, respectivamente (Tabela 1). Os tratamentos que não apresentaram qualquer sintoma visual de intoxicação foram atrazine, atrazine + permethrin, atrazine + methomyl e atrazine + chlorpyrifos ethyl, igualando-se à testemunha não tratada. O grupo que apresentou os sintomas mais intensos foi composto por nicosulfuron + chlorpyrifos ethyl, mesotrione + chlorpyrifos ethyl e 2,4-D + chlorpyrifos ethyl. Entretanto, destaca-se que a intensidade dos sintomas foi baixa, pois em nenhum tratamento o nível de intoxicação foi superior a $15 \%$.

Para as variáveis teor de clorofila, número médio de folhas por planta e massa seca da parte aérea houve o agrupamento das médias em dois grupos (Tabela 1). O teor de clorofila foi significativamente reduzido pelos tratamentos com foramsulfuron + iodosulfuron + chlorpyrifos ethyl, carfentrazone (exceto na sua mistura com methomyl), 2,4-D e 2,4-D + methomyl. Os tratamentos que mais prejudicaram o número médio de folhas por planta foram atrazine + methomyl, atrazine + chlorpyrifos ethyl, nicosulfuron + chlorpyrifos ethyl, foramsulfuron + iodosulfuron (exceto na mistura com novarulon), mesotrione (exceto mistura com novarulon), carfentrazone + methomyl e 2,4-D (exceto misturas com permethrin e methomyl). O grupo de tratamentos que causaram redução na massa seca da parte aérea foi composto por nicosulfuron + chlorpyrifos ethyl, foramsulfuron + iodosulfuron, foramsulfuron + iodosulfuron + per- 
Tabela 1. Intoxicação visual (INTO, \%), altura (ALT, cm), teor de clorofila (índice SPAD), número de folhas (NFL), massa seca da parte aérea (MSPA) e de raízes (MSR) de plantas de milho híbrido 30F35, aos 7 e 14 DAA (dias após aplicação) no estádio fenológico $\mathrm{V}_{3}$.

\begin{tabular}{|c|c|c|c|c|c|c|c|}
\hline \multirow{3}{*}{ Tratamentos } & \multicolumn{7}{|c|}{ Híbrido $30 \mathrm{~F} 35$ - Estádio $V_{3}$} \\
\hline & INTO & INTO & ALT & SPAD & NFL & MSPA & MSR \\
\hline & 7DAA & 14DAA & 14DAA & 14 DAA & 14DAA & 14DAA & $14 \mathrm{DAA}$ \\
\hline 1. atrazine ${ }^{/ 1}$ & $0,0 \mathrm{f}$ & $0,0 \mathrm{~g}$ & $35,6 \mathrm{a}$ & $36,3 \mathrm{a}$ & $7,6 \mathrm{a}$ & $1,80 \mathrm{a}$ & $0,58 \mathrm{a}$ \\
\hline 2. atrazine + permethrin ${ }^{17}$ & $0,0 \mathrm{f}$ & $0,0 \mathrm{~g}$ & $33,5 \mathrm{a}$ & $36,4 \mathrm{a}$ & $7,6 \mathrm{a}$ & $1,83 \mathrm{a}$ & $0,45 \mathrm{a}$ \\
\hline 3. atrazine + methomy ${ }^{18}$ & $0,0 \mathrm{f}$ & $0,0 \mathrm{~g}$ & $36,9 \mathrm{a}$ & $35,8 \mathrm{a}$ & $7,2 \mathrm{~b}$ & $1,79 \mathrm{a}$ & $0,40 \mathrm{~b}$ \\
\hline 4. atrazine + chlorpyrifos ethyl ${ }^{19}$ & $0,0 \mathrm{f}$ & $0,0 \mathrm{~g}$ & $36,7 \mathrm{a}$ & $36,4 \mathrm{a}$ & $7,4 \mathrm{~b}$ & $1,93 \mathrm{a}$ & $0,31 \mathrm{c}$ \\
\hline 5. atrazine + novarulon $^{/ 10}$ & $3,0 \mathrm{e}$ & $3,0 \mathrm{f}$ & $33,6 \mathrm{a}$ & $34,7 \mathrm{a}$ & $7,6 \mathrm{a}$ & $1,95 \mathrm{a}$ & $0,43 \mathrm{~b}$ \\
\hline 6. nicosulfuron ${ }^{2}$ & $5,0 \mathrm{~d}$ & $5,0 \mathrm{e}$ & $33,9 \mathrm{a}$ & $34,3 \mathrm{a}$ & $7,6 \mathrm{a}$ & $1,88 \mathrm{a}$ & $0,43 \mathrm{~b}$ \\
\hline 7. nicosulfuron + permethrin & $5,8 \mathrm{~d}$ & $3,8 \mathrm{e}$ & $34,9 \mathrm{a}$ & $36,6 \mathrm{a}$ & $8,2 \mathrm{a}$ & $2,12 \mathrm{a}$ & $0,29 \mathrm{c}$ \\
\hline 8. nicosulfuron + methomyl & $5,4 \mathrm{~d}$ & $4,6 \mathrm{e}$ & $33,6 \mathrm{a}$ & $35,4 \mathrm{a}$ & $7,6 \mathrm{a}$ & $1,89 \mathrm{a}$ & $0,34 \mathrm{~b}$ \\
\hline 9. nicosulfuron + chlorpyrifos ethyl & $14,4 \mathrm{a}$ & $11,6 \mathrm{~b}$ & $30,3 \mathrm{a}$ & $34,6 \mathrm{a}$ & $7,0 \mathrm{~b}$ & $1,01 \mathrm{~b}$ & $0,23 \mathrm{c}$ \\
\hline 10. nicosulfuron + novarulon & $7,6 \mathrm{c}$ & $5,0 \mathrm{e}$ & $32,1 \mathrm{a}$ & $35,1 \mathrm{a}$ & $7,6 \mathrm{a}$ & $1,77 \mathrm{a}$ & $0,46 \mathrm{a}$ \\
\hline 11. foramulfuron + iodosulfuron ${ }^{1 / 3}$ & $4,2 \mathrm{e}$ & $6,4 \mathrm{~d}$ & $38,0 \mathrm{a}$ & $35,0 \mathrm{a}$ & $7,0 \mathrm{~b}$ & $1,58 \mathrm{~b}$ & $0,17 \mathrm{c}$ \\
\hline 12. foram + iodo + permethrin & $7,8 \mathrm{c}$ & $4,6 \mathrm{e}$ & $34,3 \mathrm{a}$ & $35,8 \mathrm{a}$ & $7,4 \mathrm{~b}$ & $1,33 \mathrm{~b}$ & $0,17 \mathrm{c}$ \\
\hline 13. foram + iodo + methomyl & $6,8 \mathrm{~d}$ & $5,6 \mathrm{~d}$ & $34,8 \mathrm{a}$ & 33,6 a & $7,0 \mathrm{~b}$ & $1,57 \mathrm{~b}$ & $0,22 \mathrm{c}$ \\
\hline 14. foram + iodo + chlorpyrifos ethyl & $12,4 \mathrm{~b}$ & $9,2 \mathrm{c}$ & $29,6 \mathrm{a}$ & $31,2 \mathrm{~b}$ & $7,0 \mathrm{~b}$ & $1,73 \mathrm{a}$ & $0,22 \mathrm{c}$ \\
\hline 15. foram + iodo + novarulon & $6,8 \mathrm{~d}$ & $7,2 \mathrm{~d}$ & $32,3 \mathrm{a}$ & $33,5 \mathrm{a}$ & $8,0 \mathrm{a}$ & $1,90 \mathrm{a}$ & $0,23 \mathrm{c}$ \\
\hline 16. mesotrione ${ }^{/ 4}$ & $3,4 \mathrm{e}$ & $6,0 \mathrm{~d}$ & $29,6 \mathrm{a}$ & $35,4 \mathrm{a}$ & $7,2 \mathrm{~b}$ & $1,60 \mathrm{~b}$ & $0,50 \mathrm{a}$ \\
\hline 17. mesotrione + permethrin & $3,4 \mathrm{e}$ & $6,0 \mathrm{~d}$ & $34,6 \mathrm{a}$ & $33,9 \mathrm{a}$ & $7,4 \mathrm{~b}$ & $1,75 \mathrm{a}$ & $0,33 \mathrm{~b}$ \\
\hline 18. mesotrione + methomyl & $3,8 \mathrm{e}$ & $6,2 \mathrm{~d}$ & $35,5 \mathrm{a}$ & $34,2 \mathrm{a}$ & $7,0 \mathrm{~b}$ & $1,24 \mathrm{~b}$ & $0,37 \mathrm{~b}$ \\
\hline 19. mesotrione + chlorpyrifos ethyl & $14,4 \mathrm{a}$ & $13,8 \mathrm{a}$ & $34,6 \mathrm{a}$ & 33,8 a & $7,4 \mathrm{~b}$ & $1,28 \mathrm{~b}$ & $0,27 \mathrm{c}$ \\
\hline 20. mesotrione + novarulon & $5,2 \mathrm{~d}$ & $7,2 \mathrm{~d}$ & $35,7 \mathrm{a}$ & 33,4 a & $7,6 \mathrm{a}$ & $1,63 \mathrm{~b}$ & $0,35 \mathrm{~b}$ \\
\hline 21. carfentrazone ${ }^{/ 5}$ & $5,4 \mathrm{~d}$ & $5,0 \mathrm{e}$ & $35,9 \mathrm{a}$ & $29,9 \mathrm{~b}$ & $7,8 \mathrm{a}$ & $2,28 \mathrm{a}$ & $0,35 \mathrm{~b}$ \\
\hline 22. carfentrazone + permethrin & $5,4 \mathrm{~d}$ & $3,8 \mathrm{e}$ & $35,6 \mathrm{a}$ & $30,6 \mathrm{~b}$ & $7,8 \mathrm{a}$ & $2,18 \mathrm{a}$ & $0,18 \mathrm{c}$ \\
\hline 23. carfentrazone + methomyl & $5,4 \mathrm{~d}$ & $4,2 \mathrm{e}$ & $33,0 \mathrm{a}$ & $33,9 \mathrm{a}$ & $7,4 \mathrm{~b}$ & $2,02 \mathrm{a}$ & $0,39 \mathrm{~b}$ \\
\hline 24. carfentrazone + chlorpyrifos ethyl & $11,2 \mathrm{~b}$ & $6,2 \mathrm{~d}$ & $32,3 \mathrm{a}$ & $30,7 \mathrm{~b}$ & $8,0 \mathrm{a}$ & $1,58 \mathrm{~b}$ & $0,20 \mathrm{c}$ \\
\hline 25. carfentrazone + novarulon & $5,8 \mathrm{~d}$ & $5,0 \mathrm{e}$ & $36,2 \mathrm{a}$ & $31,6 \mathrm{~b}$ & $8,0 \mathrm{a}$ & $2,20 \mathrm{a}$ & $0,23 \mathrm{c}$ \\
\hline $26.2,4-\mathrm{D}^{/ 6}$ & $9,2 \mathrm{c}$ & $6,8 \mathrm{~d}$ & $32,6 \mathrm{a}$ & $32,8 \mathrm{~b}$ & $7,4 \mathrm{~b}$ & $1,47 \mathrm{~b}$ & $0,36 \mathrm{~b}$ \\
\hline 27. 2,4-D + permethrin & $9,4 \mathrm{c}$ & $6,2 \mathrm{~d}$ & $33,1 \mathrm{a}$ & $32,3 \mathrm{~b}$ & $7,8 \mathrm{a}$ & $1,49 \mathrm{~b}$ & $0,42 \mathrm{~b}$ \\
\hline 28. 2,4-D + methomyl & $12,0 \mathrm{~b}$ & $5,6 \mathrm{~d}$ & $32,2 \mathrm{a}$ & $31,8 \mathrm{~b}$ & $7,6 \mathrm{a}$ & $1,51 \mathrm{~b}$ & $0,43 \mathrm{~b}$ \\
\hline 29. 2,4-D + chlorpyrifos ethyl & $12,2 \mathrm{a}$ & $5,6 \mathrm{~d}$ & $32,5 \mathrm{a}$ & $32,5 \mathrm{~b}$ & $7,2 \mathrm{~b}$ & $1,22 \mathrm{~b}$ & $0,34 \mathrm{~b}$ \\
\hline 30. 2,4-D + novarulon & $11,6 b$ & $6,8 \mathrm{~d}$ & $30,3 \mathrm{a}$ & $30,6 \mathrm{~b}$ & $7,4 b$ & $1,44 b$ & $0,35 \mathrm{~b}$ \\
\hline 31. testemunha sem aplicação & $0,0 \mathrm{f}$ & $0,0 \mathrm{~g}$ & $35,6 \mathrm{a}$ & $35,4 \mathrm{a}$ & $8,0 \mathrm{a}$ & $2,23 \mathrm{a}$ & $0,54 \mathrm{a}$ \\
\hline $\bar{F}$ & $91,87^{*}$ & $49,34 *$ & $23,29^{\mathrm{ns}}$ & $19,07 *$ & $0,57^{*}$ & $0,52 *$ & $0,06^{*}$ \\
\hline C.V. $(\%)$ & 25,80 & 24,41 & 11,56 & 5,95 & 6,86 & 21,67 & 34,33 \\
\hline
\end{tabular}

${ }^{11}=$ Siptran $500 \mathrm{SC}^{\circledR} ;{ }^{12}=$ Accent $^{\circledR} ;{ }^{13}=$ Equip Plus $^{\circledR} ;{ }^{14}=$ Callisto $^{\circledR} ;{ }^{15}=$ Aurora $400 \mathrm{CE}^{\circledR} ;{ }^{16}=$ DMA $^{8} 06 \mathrm{BR}^{\circledR} ;{ }^{17}=$ Piredan $^{\circledR} ;{ }^{18}=$ Lannate $\mathrm{BR}^{\circledR} ; /{ }^{19}=$ Lorsban $480 \mathrm{BR}^{\circledR} ; / 10=$ Gallaxy $100 \mathrm{EC}^{\circledR}$. Médias seguidas de mesma letra na coluna não diferem entre si pelo teste de agrupamento de Scott-Knott, em nível de 5\% de probabilidade. $(*$ = significativo; ns = não significativo) 
methrin, foramsulfuron + iodosulfuron + methomyl, mesotrione (exceto mistura com permethrin), carfentyrazone + chlorpyrifos ethyl e 2,4-D.

A massa seca das raízes do híbrido $30 \mathrm{~F} 35$ foi significativamente reduzida pela maioria dos herbicidas e associações com inseticidas (Tabela 1), resultando em um pequeno agrupamento de tratamentos que não afetaram esta variável, a saber, atrazine, atrazine + permethrin, nicosulfuron + novarulon e mesotrione. Nesse sentido, uma analise geral permite observar que apenas as misturas atrazine + permethrin e nicosulfuon + novarulon aplicadas em $\mathrm{V}_{3}$ não afetaram nenhuma variável ligada ao crescimento inicial do híbrido 30F35. Por outro lado, as misturas em tanque entre nicosulfuron + chlorpyrifos ethyl, mesotrione + chlorpyrifos ethyl e 2,4-D + chlorpyrifos ethyl afetaram a maioria das variáveis avaliadas.

Ao contrário do estádio $\mathrm{V}_{3}$, a aplicação dos tratamentos em $\mathrm{V}_{6}$ afetou significativamente um número maior de variáveis avaliadas para o híbrido 30F35 (Tabela 2). Esse resultado reforça a ideia de que a aplicação tardia, como a realizada em $\mathrm{V}_{6}$, pode influenciar a sensibilidade do híbrido, tendo sido, de forma geral, mais prejudicial. Esses resultados corroboram os de Spader e Vidal (2001) e Cavalieri et al. (2012) que relataram o nicosulfuron sendo mais seletivo aos híbridos de milho comum AG5011 e pipoca $A 448$ White em estádio $V_{3}$, ao contrário de $\mathrm{V}_{6}, \mathrm{~V}_{7}$ e $\mathrm{V}_{9}$, os quais reduziram significativamente $\mathrm{o}$ rendimento da cultura. López-Ovejero et al. (2003) também observaram redução no rendimento do milho para aplicação de herbicidas após o estádio $\mathrm{V}_{4}$. Segundo Spader e Vidal (2001), a maior sensibilidade dos híbridos de milho em estádio mais avançado de desenvolvimento deve-se à maior interceptação do herbicida na aplicação, e à maior área foliar fotossinteticamente ativa, produzindo assim maior quantida- de de fotoassimilados, que são transportados para as regiões de maior demanda da planta. Desta forma, o herbicida absorvido é translocado em maior intensidade nas plantas mais desenvolvidas para os locais de ação antes da metabolização.

Os tratamentos compostos por atrazine, atrazine + permethrin, atrazine + metomyl e atrazine + chlorpyrifos ethyl não apresentaram sintomas visuais de intoxicação aos 7 e 14 DAA, sendo agrupados com a testemunha não tratada (Tabela 2). Esses resultados corroboram os de Trezzi et al. (2005), os quais não observaram sintomas de injúrias em plantas do híbrido AG3010 com a mistura de atrazine + chlorpyrifos ethyl $\left(3000+225 \mathrm{~g} \mathrm{ha}^{-1}\right)$. De forma contrária, os maiores níveis de intoxicação foram observados com foramsulfuron + iodosulfuron + chlorpyrifos ethyl, 2,4-D + methomyl e 2,4-D + chlorpyrifos ethyl, com níveis de injúrias oscilando entre 17,6 e 18,8\% aos 14 DAA. Trezzi et al. (2005) relataram aumento de $150 \%$ na toxicidade da mistura de foramsulfuron + iodosulfuron + chlorpyrifos ethyl $\left(45+3+225 \mathrm{~g} \mathrm{ha}^{-1}\right)$ para o híbrido AG3010, quando submetido a aplicação realizada em $V_{9}$ em relação ao $V_{7}$.

A interação entre inseticidas organofosforados, tais como o chlorpyrifos ethyl, e herbicidas do grupo das sulfonilureias, é conhecida por ser antagônica para a cultura do milho. Portanto, recomenda-se cautela, uma vez que inúmeros fatores podem afetar negativamente essa interação como método de aplicação, formulações, híbridos e disponibilidade hídrica (Silva et al., 2005). Nicolai et al. (2006) constataram intoxicação para o híbrido AGN 2012 na ordem de 27,5 e $22,5 \%$ utilizando associações de nicosulfuron + atrazine $\left(20+1500 \mathrm{~g} \mathrm{ha}^{-1}\right)$ com chlorpyrifos ethyl proveniente de formulações concentrado emulsionável (CE $240 \mathrm{~g} \mathrm{ha}^{-1}$ ) e emulsão concentrada (EC $225 \mathrm{~g}$ ha $\left.^{-1}\right)$, respectivamente. 
Tabela 2. Intoxicação visual (INTO, \%), altura (ALT, cm), teor de clorofila (índice SPAD), número de folhas (NFL), massa seca da parte aérea (MSPA) e de raízes (MSR) de plantas de milho híbrido 30F35, aos 7 e 14 DAA (dias após aplicação) no estádio fenológico $\mathrm{V}_{6}$.

\begin{tabular}{|c|c|c|c|c|c|c|c|}
\hline \multirow{3}{*}{ Tratamentos } & \multicolumn{7}{|c|}{ Híbrido $30 \mathrm{~F} 35$ - Estádio $\mathrm{V}_{6}$} \\
\hline & INTO & INTO & ALT & SPAD & NFL & MSPA & MSR \\
\hline & 7DAA & 14DAA & 14DAA & 14 DAA & 14DAA & 14DAA & $14 \mathrm{DAA}$ \\
\hline 1. atrazine $^{/ 1}$ & $0,0 \mathrm{f}$ & $0,0 \mathrm{~h}$ & $64,8 \mathrm{a}$ & $34,5 \mathrm{a}$ & $10,6 \mathrm{a}$ & $15,63 \mathrm{a}$ & $2,95 \mathrm{~b}$ \\
\hline 2. atrazine + permethrin ${ }^{17}$ & $0,0 \mathrm{f}$ & $0,0 \mathrm{~h}$ & $65,4 \mathrm{a}$ & $34,0 \mathrm{a}$ & $11,8 \mathrm{a}$ & $14,90 \mathrm{a}$ & $2,85 \mathrm{~b}$ \\
\hline 3. atrazine + methomy ${ }^{18}$ & $0,0 \mathrm{f}$ & $0,0 \mathrm{~h}$ & $65,0 \mathrm{a}$ & $34,6 \mathrm{a}$ & $11,4 \mathrm{a}$ & $15,00 \mathrm{a}$ & $2,28 \mathrm{c}$ \\
\hline 4. atrazine + chlorpyrifos ethyl $^{19}$ & $0,0 \mathrm{f}$ & $0,0 \mathrm{~h}$ & $69,7 \mathrm{a}$ & $37,8 \mathrm{a}$ & $11,2 \mathrm{a}$ & $14,00 \mathrm{~b}$ & $2,19 \mathrm{c}$ \\
\hline 5. atrazine + novarulon $^{/ 10}$ & $3,0 \mathrm{f}$ & $3,0 \mathrm{~g}$ & 64,9 a & $32,7 \mathrm{~b}$ & $11,0 \mathrm{a}$ & 15,17 a & $2,81 \mathrm{~b}$ \\
\hline 6. nicosulfuron ${ }^{7}$ & $7,4 \mathrm{e}$ & $6,2 \mathrm{f}$ & $63,7 \mathrm{a}$ & $32,4 \mathrm{~b}$ & $11,4 \mathrm{a}$ & $14,83 \mathrm{a}$ & $2,60 \mathrm{~b}$ \\
\hline 7. nicosulfuron + permethrin & $12,6 \mathrm{~d}$ & $6,2 \mathrm{f}$ & $67,0 \mathrm{a}$ & $35,2 \mathrm{a}$ & $11,6 \mathrm{a}$ & $15,20 \mathrm{a}$ & $1,96 \mathrm{c}$ \\
\hline 8. nicosulfuron + methomyl & $15,0 \mathrm{c}$ & $6,2 \mathrm{f}$ & $65,5 \mathrm{a}$ & $33,4 \mathrm{a}$ & $11,6 \mathrm{a}$ & $15,93 \mathrm{a}$ & $1,98 \mathrm{c}$ \\
\hline 9. nicosulfuron + chlorpyrifos ethyl & $19,8 \mathrm{~b}$ & $9,2 \mathrm{e}$ & $65,0 \mathrm{a}$ & $32,2 \mathrm{~b}$ & $11,4 \mathrm{a}$ & $13,66 \mathrm{~b}$ & $2,52 b$ \\
\hline 10. nicosulfuron + novarulon & $17,6 \mathrm{~b}$ & $6,2 \mathrm{f}$ & $67,3 \mathrm{a}$ & $33,0 \mathrm{~b}$ & $11,6 \mathrm{a}$ & $14,92 \mathrm{a}$ & $2,51 \mathrm{~b}$ \\
\hline 11. foramulfuron + iodosulfuron ${ }^{/ 3}$ & $15,0 \mathrm{c}$ & $8,2 \mathrm{e}$ & $64,4 \mathrm{a}$ & $34,1 \mathrm{a}$ & $11,8 \mathrm{a}$ & $15,49 \mathrm{a}$ & $2,33 \mathrm{c}$ \\
\hline 12. foram + iodo + permethrin & $18,8 \mathrm{~b}$ & $8,4 \mathrm{e}$ & $61,4 \mathrm{~b}$ & $34,0 \mathrm{a}$ & $11,2 \mathrm{a}$ & $13,13 \mathrm{~b}$ & $2,30 \mathrm{c}$ \\
\hline 13. foram + iodo + methomyl & $19,4 \mathrm{~b}$ & $9,8 \mathrm{e}$ & $58,8 \mathrm{~b}$ & $32,4 \mathrm{~b}$ & $10,6 \mathrm{a}$ & $11,50 \mathrm{c}$ & $1,84 \mathrm{c}$ \\
\hline 14. foram + iodo + chlorpyrifos ethyl & $23,2 \mathrm{a}$ & $18,6 \mathrm{a}$ & $52,4 \mathrm{c}$ & $29,0 \mathrm{c}$ & $10,4 \mathrm{~b}$ & $9,41 \mathrm{~d}$ & $1,53 \mathrm{c}$ \\
\hline 15. foram + iodo + novarulon & $18,4 \mathrm{~b}$ & $8,4 \mathrm{e}$ & $68,5 \mathrm{a}$ & $31,9 \mathrm{~b}$ & $11,4 \mathrm{a}$ & $12,88 \mathrm{c}$ & $1,92 \mathrm{c}$ \\
\hline 16. mesotrione ${ }^{1 / 4}$ & $4,6 \mathrm{e}$ & $7,8 \mathrm{f}$ & $60,3 \mathrm{~b}$ & $32,5 \mathrm{~b}$ & $11,6 \mathrm{a}$ & $15,64 \mathrm{a}$ & $3,77 \mathrm{a}$ \\
\hline 17. mesotrione + permethrin & $5,8 \mathrm{e}$ & $9,2 \mathrm{e}$ & $62,4 \mathrm{~b}$ & $32,4 \mathrm{~b}$ & $11,0 \mathrm{a}$ & $15,14 \mathrm{a}$ & $2,24 \mathrm{c}$ \\
\hline 18. mesotrione + methomyl & $5,6 \mathrm{e}$ & $9,2 \mathrm{e}$ & $68,1 \mathrm{a}$ & $33,0 \mathrm{~b}$ & $11,0 \mathrm{a}$ & $15,90 \mathrm{a}$ & $1,93 \mathrm{c}$ \\
\hline 19. mesotrione + chlorpyrifos ethyl & $12,2 \mathrm{~d}$ & $16,6 \mathrm{~b}$ & $63,4 \mathrm{a}$ & $32,2 \mathrm{~b}$ & $12,2 \mathrm{a}$ & $13,76 \mathrm{~b}$ & $1,62 \mathrm{c}$ \\
\hline 20. mesotrione + novarulon & $7,4 \mathrm{e}$ & $11,6 \mathrm{~d}$ & $63,8 \mathrm{a}$ & $32,3 \mathrm{~b}$ & $11,8 \mathrm{a}$ & 16,19 a & $2,53 \mathrm{~b}$ \\
\hline 21. carfentrazone ${ }^{15}$ & $13,0 \mathrm{~d}$ & $6,8 \mathrm{f}$ & $58,1 \mathrm{~b}$ & $30,0 \mathrm{c}$ & $12,0 \mathrm{a}$ & $14,14 \mathrm{~b}$ & $2,03 \mathrm{c}$ \\
\hline 22. carfentrazone + permethrin & $15,0 \mathrm{c}$ & $6,2 \mathrm{f}$ & $59,4 \mathrm{~b}$ & $30,1 \mathrm{c}$ & $11,4 \mathrm{a}$ & $13,33 \mathrm{~b}$ & $1,89 \mathrm{c}$ \\
\hline 23. carfentrazone + methomyl & $11,6 \mathrm{~d}$ & $6,8 \mathrm{f}$ & 63,3 a & $32,0 \mathrm{~b}$ & $11,4 \mathrm{a}$ & $14,46 \mathrm{~b}$ & $2,62 \mathrm{~b}$ \\
\hline 24. carfentrazone + chlorpyrifos ethyl & $19,6 \mathrm{~b}$ & $10,6 \mathrm{e}$ & $62,2 \mathrm{~b}$ & $29,4 \mathrm{c}$ & $11,2 \mathrm{a}$ & $9,80 \mathrm{~d}$ & $1,01 \mathrm{c}$ \\
\hline 25. carfentrazone + novarulon & $14,0 \mathrm{c}$ & $7,6 \mathrm{f}$ & $60,2 \mathrm{~b}$ & $30,8 \mathrm{c}$ & $12,0 \mathrm{a}$ & $12,25 \mathrm{c}$ & $1,86 \mathrm{c}$ \\
\hline $26.2,4-\mathrm{D}^{76}$ & $11,6 \mathrm{~d}$ & $15,6 \mathrm{~b}$ & $63,3 \mathrm{a}$ & $31,0 \mathrm{c}$ & $10,0 \mathrm{c}$ & $10,75 \mathrm{~d}$ & $1,84 \mathrm{c}$ \\
\hline 27. 2,4-D + permethrin & $11,6 \mathrm{~d}$ & $14,6 \mathrm{c}$ & $60,2 \mathrm{~b}$ & $30,7 \mathrm{c}$ & $9,6 \mathrm{c}$ & $11,89 \mathrm{c}$ & $2,15 \mathrm{c}$ \\
\hline 28. 2,4-D + methomyl & $14,6 \mathrm{c}$ & $17,6 \mathrm{a}$ & 66,8 a & $30,2 \mathrm{c}$ & $9,8 \mathrm{c}$ & $10,41 \mathrm{~d}$ & $1,75 \mathrm{c}$ \\
\hline 29. 2,4-D + chlorpyrifos ethyl & $15,6 \mathrm{c}$ & $18,8 \mathrm{a}$ & $68,2 \mathrm{a}$ & $30,6 \mathrm{c}$ & $9,4 \mathrm{c}$ & $11,83 \mathrm{c}$ & $1,91 \mathrm{c}$ \\
\hline 30. 2,4-D + novarulon & $15,6 \mathrm{c}$ & $16,6 \mathrm{~b}$ & $69,6 \mathrm{a}$ & $29,6 \mathrm{c}$ & $9,4 \mathrm{c}$ & $11,97 \mathrm{c}$ & $1,87 \mathrm{c}$ \\
\hline 31. testemunha sem aplicação & $0,0 \mathrm{f}$ & $0,0 \mathrm{~h}$ & $70,0 \mathrm{a}$ & $33,7 \mathrm{a}$ & $12,0 \mathrm{a}$ & $15,74 \mathrm{a}$ & $3,97 \mathrm{a}$ \\
\hline $\bar{F}$ & $240,30 *$ & $158,64^{*}$ & $77,35^{*}$ & $14,82 *$ & $3,10^{*}$ & $19,19^{*}$ & $1,92 *$ \\
\hline C.V. $(\%)$ & 19,97 & 22,09 & 7,06 & 5,20 & 7,09 & 11,79 & 22,44 \\
\hline
\end{tabular}

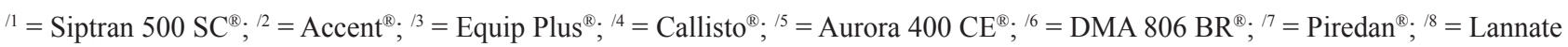
$\mathrm{BR}^{\circledR} ;{ }^{19}=$ Lorsban $480 \mathrm{BR}^{\circledR} ;{ }^{10}=$ Gallaxy $100 \mathrm{EC}^{\circledR}$. Médias seguidas de mesma letra na coluna não diferem entre si pelo teste de agrupamento de Scott-Knott, em nível de 5\% de probabilidade. $(*$ significativo; ns = não significativo) 
Relatos de injúrias acentuadas na cultura do milho são mais comumente encontradas na literatura para misturas de inseticidas organofosforados com herbicidas inibidores de ALS, e tem como a principal causa a inibição de enzimas do citocromo P450, que proporciona redução na metabolização dos herbicidas (Koeppe et al., 2000). Segundo Diehl et al. (1995), esta maior toxicidade, mesmo em híbridos tolerantes, também pode ocorrer devido à redução de cera epicuticular causada pelo inseticida e, consequentemente, aumento da absorção dos herbicidas.

Para as variáveis altura de plantas, teor de clorofila, número médio de folhas por planta e massa seca das raízes houve o agrupamento das médias em três grupos pelo teste de Scott-Knott. Para a variável massa seca da parte aérea foram formados quatro grupos de médias (Tabela 2). Alguns tratamentos se destacaram pela constância dos efeitos nocivos ao crescimento inicial do híbrido 30F35, como observado para a mistura em tanque de foramsulfuron + iodosulfuron + chlorpyrifos ethyl e as misturas envolvendo o herbicida 2,4-D, que sempre estiveram entre os tratamentos mais nocivos na aplicação em estádio $\mathrm{V}_{6}$. Trezzi et al. (2005) também relataram redução significativa na altura de plantas de milho com a mistura foramsulfuron + iodosulfuron + chlorpyrifos ethyl aos 15 DAA. Silva et al. (2007) constaram redução significativamente da massa seca da cultura do milho para mistura em tanque de atrazine + nicosulfuron + chlorpyrifos ethyl aos 60 DAA.

De maneira contrária ao que foi descrito para a aplicação em $\mathrm{V}_{3}$, não se observou nenhum tratamento com resultados semelhantes à testemunha não tratada, em todas as variáveis avaliadas. Esses resultados confirmam que a aplicação de tratamentos envolvendo mistura em tanque entre herbicidas e inseticidas para o híbrido $30 \mathrm{~F} 35$ foram mais prejudiciais quando realizadas em estádio $\mathrm{V}_{6}$. As misturas em tanque entre os herbicidas do grupo químico das sulfonilureias (nicosulfuron ou foramsulfuron + iodosulfuron) ou 2,4-D com o inseticida organofosforado chlorpyrifos ethyl estiveram entre os tratamentos que mais prejudicaram o crescimento inicial do híbrido 30F35, independentemente do estádio de aplicação.

\section{Experimento 2: híbrido 2B-710}

A aplicação no estádio $\mathrm{V}_{3}$ não resultou em intoxicação visual das plantas de milho nos tratamentos envolvendo atrazine, nicosulfuron, foramsulfuron + iodosulfuron e mesotrione, exceto para as misturas dos três últimos com chlorpyrifos ethyl (Tabela 3). Por outro lado, alguns tratamentos causaram elevada intoxicação, com destaque para carfentrazone + chlorpyrifos ethyl e mesotrione + chlorpyrifos ethyl, os quais provocaram níveis de injúrias de 45,6 e $66,0 \%$ aos 7 DAA e de 17,6 e 44,0\% aos 14 DAA, respectivamente. De forma contrária, Nicolai et al. (2006) e Steckel et al. (2015) observaram baixos níveis de intoxicação e nenhum efeito para as misturas de mesotrione + atrazine e mesotrione + s-metolachlor + glyphosate com chlorpyrifos ethyl sobre a produtividade dos híbridos de milho AGN 2012 e DKC6188 VT Triplo Pro, quando aplicadas nos estádios $\mathrm{V}_{4-5} \mathrm{e}$ $\mathrm{V}_{3}$, respectivamente. Segundo Ogliari et al. (2014), alguns híbridos de milho são mais sensíveis ao mesotrione. Todavia, o mecanismo de tolerância do milho a esse herbicida ainda é desconhecido.

A altura de plantas e o teor de clorofila foram afetados em diferentes níveis pelos tratamentos, com agrupamento dos resultados em quatro grupos. As misturas envolvendo nicosulfuron, foramsulfuron + iodosulfuron, mesotrione ou carfentrazone com o inseticida chlorpyrifos ethyl foram agrupadas entre as que 
Tabela 3. Intoxicação visual (INTO, \%), altura (ALT, cm), teor de clorofila (índice SPAD), número de folhas (NFL), massa seca da parte aérea (MSPA) e de raízes (MSR) de plantas de milho híbrido 2B-710, aos 7 e 14 DAA (dias após aplicação) no estádio fenológico $\mathrm{V}_{3}$.

\begin{tabular}{|c|c|c|c|c|c|c|c|}
\hline \multirow{3}{*}{ Tratamentos } & \multicolumn{7}{|c|}{ Híbrido 2B-710 - Estádio $V_{3}$} \\
\hline & INTO & INTO & ALT & SPAD & NFL & MSPA & MSR \\
\hline & 7DAA & 14DAA & 14DAA & 14 DAA & 14DAA & 14DAA & $14 \mathrm{DAA}$ \\
\hline 1. atrazine $^{/ 1}$ & $0,0 \mathrm{~g}$ & $0,0 \mathrm{f}$ & $34,0 \mathrm{a}$ & $37,5 \mathrm{a}$ & $6,2 \mathrm{~b}$ & $1,17 \mathrm{a}$ & $0,34 \mathrm{a}$ \\
\hline 2. atrazine + permethrin ${ }^{17}$ & $0,0 \mathrm{~g}$ & $0,0 \mathrm{f}$ & $33,9 \mathrm{a}$ & $37,5 \mathrm{a}$ & $6,4 \mathrm{~b}$ & $1,16 \mathrm{a}$ & $0,38 \mathrm{a}$ \\
\hline 3. atrazine + methomy ${ }^{18}$ & $0,0 \mathrm{~g}$ & $0,0 \mathrm{f}$ & $31,4 \mathrm{a}$ & $38,2 \mathrm{a}$ & $6,0 \mathrm{~b}$ & $1,00 \mathrm{a}$ & $0,29 \mathrm{a}$ \\
\hline 4. atrazine + chlorpyrifos ethyl ${ }^{/ 9}$ & $0,0 \mathrm{~g}$ & $0,0 \mathrm{f}$ & $32,6 \mathrm{a}$ & $36,5 \mathrm{a}$ & $6,0 \mathrm{~b}$ & $1,08 \mathrm{a}$ & $0,30 \mathrm{a}$ \\
\hline 5. atrazine + novarulon $/ 10$ & $0,0 \mathrm{~g}$ & $0,0 \mathrm{f}$ & $33,5 \mathrm{a}$ & $37,1 \mathrm{a}$ & $6,0 \mathrm{~b}$ & $0,99 \mathrm{a}$ & $0,35 \mathrm{a}$ \\
\hline 6. nicosulfuron ${ }^{2}$ & $3,0 \mathrm{f}$ & $0,0 \mathrm{f}$ & $32,1 \mathrm{a}$ & $35,0 \mathrm{~b}$ & $6,4 \mathrm{~b}$ & $1,15 \mathrm{a}$ & $0,35 \mathrm{a}$ \\
\hline 7. nicosulfuron + permethrin & $0,0 \mathrm{~g}$ & $0,0 \mathrm{f}$ & $32,4 \mathrm{a}$ & $33,9 \mathrm{~b}$ & $6,4 \mathrm{~b}$ & $1,16 \mathrm{a}$ & $0,38 \mathrm{a}$ \\
\hline 8. nicosulfuron + methomyl & $0,0 \mathrm{~g}$ & $0,0 \mathrm{f}$ & $32,3 \mathrm{a}$ & $34,7 \mathrm{~b}$ & $7,0 \mathrm{a}$ & $1,18 \mathrm{a}$ & $0,37 \mathrm{a}$ \\
\hline 9. nicosulfuron + chlorpyrifos ethyl & $25,6 \mathrm{c}$ & $8,4 \mathrm{~d}$ & $22,2 \mathrm{c}$ & $32,8 \mathrm{~b}$ & $7,0 \mathrm{a}$ & $0,73 \mathrm{c}$ & $0,22 \mathrm{~b}$ \\
\hline 10. nicosulfuron + novarulon & $3,0 \mathrm{f}$ & $3,0 \mathrm{e}$ & $30,2 \mathrm{~b}$ & $35,3 \mathrm{~b}$ & $6,8 \mathrm{a}$ & $1,10 \mathrm{a}$ & $0,32 \mathrm{a}$ \\
\hline 11. foramulfuron + iodosulfuron ${ }^{/ 3}$ & $3,4 \mathrm{f}$ & $0,0 \mathrm{f}$ & $31,6 \mathrm{a}$ & $36,0 \mathrm{~b}$ & $6,0 \mathrm{~b}$ & $0,96 \mathrm{~b}$ & $0,33 \mathrm{a}$ \\
\hline 12. foram + iodo + permethrin & $0,0 \mathrm{~g}$ & $0,0 \mathrm{f}$ & $32,8 \mathrm{a}$ & $34,4 \mathrm{~b}$ & $6,6 \mathrm{a}$ & $1,10 \mathrm{a}$ & $0,28 \mathrm{a}$ \\
\hline 13. foram + iodo + methomyl & $3,0 \mathrm{f}$ & $0,0 \mathrm{f}$ & $32,1 \mathrm{a}$ & $34,6 \mathrm{~b}$ & $6,8 \mathrm{a}$ & $1,16 \mathrm{a}$ & $0,32 \mathrm{a}$ \\
\hline 14. foram + iodo + chlorpyrifos ethyl & $23,0 \mathrm{c}$ & $12,0 \mathrm{c}$ & $21,6 \mathrm{c}$ & $29,6 \mathrm{c}$ & $6,6 \mathrm{a}$ & $0,31 \mathrm{~d}$ & $0,21 \mathrm{~b}$ \\
\hline 15. foram + iodo + novarulon & $8,8 \mathrm{f}$ & $0,0 \mathrm{f}$ & $32,8 \mathrm{a}$ & $34,5 \mathrm{~b}$ & $6,4 \mathrm{~b}$ & $0,84 \mathrm{c}$ & $0,32 \mathrm{a}$ \\
\hline 16. mesotrione ${ }^{74}$ & $0,0 \mathrm{~g}$ & $0,0 \mathrm{f}$ & $31,3 \mathrm{a}$ & $35,7 \mathrm{~b}$ & $6,4 \mathrm{~b}$ & $1,15 \mathrm{a}$ & $0,36 \mathrm{a}$ \\
\hline 17. mesotrione + permethrin & $0,0 \mathrm{~g}$ & $0,0 \mathrm{f}$ & $32,6 \mathrm{a}$ & $36,0 \mathrm{~b}$ & $6,4 \mathrm{~b}$ & $1,16 \mathrm{a}$ & $0,34 \mathrm{a}$ \\
\hline 18. mesotrione + methomyl & $0,0 \mathrm{~g}$ & $0,0 \mathrm{f}$ & $32,3 \mathrm{a}$ & $36,5 \mathrm{a}$ & $7,0 \mathrm{a}$ & $1,15 \mathrm{a}$ & $0,31 \mathrm{a}$ \\
\hline 19. mesotrione + chlorpyrifos ethyl & $45,6 \mathrm{~b}$ & $17,6 \mathrm{~b}$ & $17,9 \mathrm{~d}$ & $31,5 \mathrm{c}$ & $6,4 \mathrm{~b}$ & $0,38 \mathrm{~d}$ & $0,13 \mathrm{~b}$ \\
\hline 20. mesotrione + novarulon & $4,8 \mathrm{f}$ & $0,0 \mathrm{f}$ & $29,8 \mathrm{~b}$ & $35,5 \mathrm{~b}$ & $6,6 \mathrm{a}$ & $1,17 \mathrm{a}$ & $0,35 \mathrm{a}$ \\
\hline 21. carfentrazone ${ }^{15}$ & $5,6 \mathrm{f}$ & $4,6 \mathrm{e}$ & $27,3 \mathrm{~b}$ & $35,1 \mathrm{~b}$ & $6,6 \mathrm{a}$ & $1,12 \mathrm{a}$ & $0,36 \mathrm{a}$ \\
\hline 22. carfentrazone + permethrin & $5,0 \mathrm{f}$ & $4,2 \mathrm{e}$ & $29,4 \mathrm{~b}$ & $35,8 \mathrm{~b}$ & $6,6 \mathrm{a}$ & $1,17 \mathrm{a}$ & $0,29 \mathrm{a}$ \\
\hline 23. carfentrazone + methomyl & $4,2 \mathrm{f}$ & $3,0 \mathrm{e}$ & $28,4 \mathrm{~b}$ & $35,8 \mathrm{~b}$ & $7,0 \mathrm{a}$ & $1,15 \mathrm{a}$ & $0,31 \mathrm{a}$ \\
\hline 24. carfentrazone + chlorpyrifos ethyl & $66,0 \mathrm{a}$ & $44,0 \mathrm{a}$ & $15,1 \mathrm{~d}$ & $23,3 \mathrm{~d}$ & $5,4 \mathrm{~b}$ & $0,12 \mathrm{e}$ & $0,16 b$ \\
\hline 25. carfentrazone + novarulon & $6,6 \mathrm{f}$ & $4,2 \mathrm{e}$ & $27,1 \mathrm{~b}$ & $35,0 \mathrm{~b}$ & $6,6 \mathrm{a}$ & $0,76 \mathrm{c}$ & $0,29 \mathrm{a}$ \\
\hline $26.2,4-\mathrm{D}^{16}$ & $20,6 \mathrm{~d}$ & $12,0 \mathrm{c}$ & $27,6 \mathrm{~b}$ & $37,4 \mathrm{a}$ & $6,4 \mathrm{~b}$ & $0,96 \mathrm{~b}$ & $0,41 \mathrm{a}$ \\
\hline 27. 2,4-D + permethrin & $20,2 \mathrm{~d}$ & $10,0 \mathrm{~d}$ & $27,1 \mathrm{~b}$ & $38,0 \mathrm{a}$ & $6,4 \mathrm{~b}$ & $1,07 \mathrm{a}$ & $0,29 \mathrm{a}$ \\
\hline 28. 2,4-D + methomyl & $20,6 \mathrm{~d}$ & $9,2 \mathrm{~d}$ & $27,6 \mathrm{~b}$ & $38,2 \mathrm{a}$ & $6,2 \mathrm{~b}$ & $0,90 \mathrm{~b}$ & $0,28 \mathrm{a}$ \\
\hline 29. 2,4-D + chlorpyrifos ethyl & $15,8 \mathrm{e}$ & $8,8 \mathrm{~d}$ & $24,6 \mathrm{c}$ & $37,7 \mathrm{a}$ & $6,0 \mathrm{~b}$ & $0,74 \mathrm{c}$ & $0,24 \mathrm{~b}$ \\
\hline 30. 2,4-D + novarulon & $14,6 \mathrm{e}$ & $7,2 \mathrm{~d}$ & $28,6 \mathrm{~b}$ & $38,3 \mathrm{a}$ & $6,2 \mathrm{~b}$ & $0,80 \mathrm{c}$ & $0,32 \mathrm{a}$ \\
\hline 31. testemunha sem aplicação & $0,0 \mathrm{~g}$ & $0,0 \mathrm{f}$ & $33,0 \mathrm{a}$ & $38,8 \mathrm{a}$ & $7,0 \mathrm{a}$ & $1,14 \mathrm{a}$ & $0,41 \mathrm{a}$ \\
\hline $\bar{F}$ & $1117,8^{*}$ & $380,7^{*}$ & $108,88^{*}$ & $45,53^{*}$ & $0,70^{*}$ & $0,38^{*}$ & $0,02 *$ \\
\hline C.V. $(\%)$ & 30,03 & 45,86 & 13,14 & 5,23 & 6,91 & 15,12 & 29,02 \\
\hline
\end{tabular}

${ }^{11}=$ Siptran $500 \mathrm{SC}^{\circledR} ;{ }^{12}=$ Accent $^{\circledR} ;{ }^{13}=$ Equip Plus $^{\circledR} ;{ }^{/ 4}=$ Callisto $^{\circledR} ;{ }^{15}=$ Aurora $400 \mathrm{CE}^{\circledR} ;{ }^{16}=$ DMA 806 BR $^{\circledR} ;{ }^{17}=$ Piredan $^{\circledR} ;{ }^{18}=$ Lannate $\mathrm{BR}^{\circledR} ;{ }^{19}=$ Lorsban $480 \mathrm{BR}^{\circledR} ; / 10=$ Gallaxy $100 \mathrm{EC}^{\circledR}$. Médias seguidas de mesma letra na coluna não diferem entre si pelo teste de agrupamento de Scott-Knott, em nível de 5\% de probabilidade. (* = significativo; ns = não significativo) 
mais afetaram negativamente estas variáveis (Tabela 3). Resultados divergentes foram observados para o número de folhas por planta, pois os tratamentos com atrazine e 2,4-D estiveram entre os mais prejudiciais.

As associações do inseticida chlorpyrifos ethyl com os herbicidas nicosulfuron, foramsulfuron + iodosulfuron, mesotrione, carfentrazone e 2,4-D aplicadas em $\mathrm{V}_{3}$ foram prejudiciais ao crescimento inicial do híbrido 2B-710, reduzindo tanto o acúmulo de massa seca na parte aérea quanto nas raízes (Tabela $3)$. Entre essas associações carfentrazone + chlorpyrifos ethyl foi a mais prejudicial ao híbrido 2B-710, sendo o tratamento menos seletivo em todas as variáveis avaliadas. Os resultados de redução de massa seca das plantas apresentaram comportamento similar ao observado nas demais variáveis de crescimento. Entretanto, em relação às misturas mais seletivas para esta modalidade de aplicação destacaram-se aquelas associadas com atrazine, uma vez que mostraram resultados semelhantes à testemunha não tratada para a maioria das variáveis analisadas.

A aplicação de atrazine isolada ou em associação com os inseticidas no estádio $\mathrm{V}_{6}$ não causou sintomas visuais de intoxicação (Tabela 4). Os tratamentos envolvendo nicosulfuron, foramsulfuron + iodosulfuron e mesotrione promoveram apenas níveis baixos de intoxicação, exceto para as misturas com chlorpyrifos ethyl. Por outro lado, os tratamentos com carfentrazone e 2,4-D causaram os níveis mais elevados de intoxicação. De forma geral, com exceção da atrazine, a mistura em tanque de chlorpyrifos ethyl aos herbicidas aplicadas no estádio $\mathrm{V}_{6}$ aumentou significativamente a toxicidade para o híbrido 2B-710 (Tabela 4). Esses resultados corroboram a afirmativa de Steckel et al. (2015) de que os principais relatos de problemas de interação antagônica entre herbicidas e inseticidas ocorrem com os organofosforados e metilcarbamatos.
Os tratamentos com maior intensidade de intoxicação mantiveram os efeitos nocivos para as variáveis altura de plantas, teor de clorofila e número médio de folhas por planta (Tabela 4). Ressalta-se que as misturas entre mesotrione + chlorpyrifos ethyl e carfentrazone + chlorpyrifos ethyl formaram o agrupamento que mais afetou negativamente o híbrido 2B-710.

As aplicações de atrazine isolada ou em associação com os inseticidas não prejudicaram o acúmulo de massa seca da parte aérea, bem como os tratamentos envolvendo os herbicidas nicosulfuron e mesotrione, exceto nas misturas com chlorpyrifos ethyl (Tabela 4). Os tratamentos com carfentrazone e 2,4-D reduziram significativamente a massa seca da parte aérea. A massa seca das raízes mostrou-se mais sensível à ação dos tratamentos estudados, sendo que apenas seis apresentaram resultados semelhantes à testemunha não tratada, a saber, atrazine, atrazine + novarulon, foramsulfuron + iodosulfuron + novarulon, mesorione + permethrin, mesotrione + methomyl e mesotrione + novarulon (Tabela 4). Destaca-se que diferentes classes de inseticidas apresentam interação com herbicidas, afetando a sua seletividade, tais como alguns carbamatos que podem ser apenas inibidores competitivos do citocromo P450. Outros inseticidas como os organofosforados, quando metabolizados pelo citocromo $\mathrm{P} 450$, liberam enxofre que se liga covalentemente à apoproteína, levando à sua inativação. Desta forma, mistura entre herbicida e inseticida que são metabolizados pelo citocromo $\mathrm{P} 450$ pode levar a cultura à morte (Werck-Reichhart et al., 2000).

Para a aplicação realizada em $\mathrm{V}_{6}$ apenas os tratamentos com atrazine isolada e mesotrione + methomyl não afetaram negativamente todas as variáveis estudadas, sendo assim consideradas altamente seletivas para o híbrido 2B-710 (Tabela 4). Por outro lado, a mistura carfentrazone + chlorpyrifos ethyl, 
Tabela 4. Intoxicação visual (INTO, \%), altura (ALT, cm), teor de clorofila (índice SPAD), número de folhas (NFL), matéria seca da parte aérea (MSPA) e de raízes (MSR) de plantas de milho híbrido 2B-710, aos 7 e 14 DAA (dias após aplicação) no estádio fenológico $\mathrm{V}_{6}$.

\begin{tabular}{|c|c|c|c|c|c|c|c|}
\hline \multirow{3}{*}{ Tratamentos } & \multicolumn{7}{|c|}{ Híbrido 2B-710 - Estádio $\mathrm{V}_{6}$} \\
\hline & INTO & INTO & $\mathrm{ALT}$ & SPAD & NFL & MSPA & MSR \\
\hline & 7DAA & 14DAA & 14DAA & 14 DAA & 14DAA & 14DAA & 14 DAA \\
\hline 1. atrazine $^{/ 1}$ & $0,0 \mathrm{f}$ & $0,0 \mathrm{~g}$ & $47,5 \mathrm{a}$ & $40,2 \mathrm{a}$ & $9,8 \mathrm{a}$ & $10,13 \mathrm{a}$ & $3,57 \mathrm{a}$ \\
\hline 2. atrazine + permethrin ${ }^{17}$ & $0,0 \mathrm{f}$ & $0,0 \mathrm{~g}$ & $47,3 \mathrm{a}$ & $39,4 \mathrm{~b}$ & $10,0 \mathrm{a}$ & $10,11 \mathrm{a}$ & $3,16 \mathrm{~b}$ \\
\hline 3. atrazine + methomy ${ }^{18}$ & $0,0 \mathrm{f}$ & $0,0 \mathrm{~g}$ & $47,4 \mathrm{a}$ & $38,6 \mathrm{~b}$ & $9,8 \mathrm{a}$ & $10,52 \mathrm{a}$ & $2,92 \mathrm{~b}$ \\
\hline 4. atrazine + chlorpyrifos ethyl ${ }^{19}$ & $0,0 \mathrm{f}$ & $0,0 \mathrm{~g}$ & $47,6 \mathrm{a}$ & $38,0 \mathrm{c}$ & $10,0 \mathrm{a}$ & $10,16 \mathrm{a}$ & $2,96 \mathrm{~b}$ \\
\hline 5. atrazine + novarulon $^{/ 10}$ & $0,0 \mathrm{f}$ & $0,0 \mathrm{~g}$ & $47,4 \mathrm{a}$ & $39,8 \mathrm{~b}$ & $10,0 \mathrm{a}$ & $10,00 \mathrm{a}$ & $3,35 \mathrm{a}$ \\
\hline 6. nicosulfuron ${ }^{2}$ & $5,8 \mathrm{e}$ & $3,4 \mathrm{~g}$ & $48,4 \mathrm{a}$ & $40,2 \mathrm{a}$ & $10,0 \mathrm{a}$ & $9,97 \mathrm{a}$ & $3,15 \mathrm{~b}$ \\
\hline 7. nicosulfuron + permethrin & $6,2 \mathrm{e}$ & $3,0 \mathrm{~g}$ & $47,6 \mathrm{a}$ & $40,8 \mathrm{a}$ & $9,8 \mathrm{a}$ & $10,76 \mathrm{a}$ & $3,00 \mathrm{~b}$ \\
\hline 8. nicosulfuron + methomyl & $11,2 \mathrm{e}$ & $3,4 \mathrm{~g}$ & 47,9 a & $40,6 \mathrm{a}$ & $10,0 \mathrm{a}$ & $10,24 \mathrm{a}$ & $3,99 \mathrm{~b}$ \\
\hline 9. nicosulfuron + chlorpyrifos ethyl & $24,4 \mathrm{c}$ & $38,0 \mathrm{~d}$ & $30,3 \mathrm{c}$ & $39,2 \mathrm{~b}$ & 9,4 a & $5,69 \mathrm{c}$ & $1,48 \mathrm{c}$ \\
\hline 10. nicosulfuron + novarulon & $7,8 \mathrm{e}$ & $3,8 \mathrm{~g}$ & $48,0 \mathrm{a}$ & $40,2 \mathrm{a}$ & $10,0 \mathrm{a}$ & $8,87 \mathrm{~b}$ & $2,96 \mathrm{~b}$ \\
\hline 11. foramulfuron + iodosulfuron ${ }^{1 / 3}$ & $6,2 \mathrm{e}$ & $0,0 \mathrm{~g}$ & $48,5 \mathrm{a}$ & $40,4 \mathrm{a}$ & $10,0 \mathrm{a}$ & $9,57 \mathrm{a}$ & $3,05 \mathrm{~b}$ \\
\hline 12. foram + iodo + permethrin & $7,2 \mathrm{e}$ & $0,0 \mathrm{~g}$ & $47,1 \mathrm{a}$ & $38,6 \mathrm{~b}$ & $9,8 \mathrm{a}$ & $7,65 \mathrm{~b}$ & $3,08 \mathrm{~b}$ \\
\hline 13. foram + iodo + methomyl & 6,8 e & $0,0 \mathrm{~g}$ & $47,1 \mathrm{a}$ & $39,6 \mathrm{~b}$ & $9,8 \mathrm{a}$ & $7,63 \mathrm{~b}$ & $2,96 \mathrm{~b}$ \\
\hline 14. foram + iodo + chlorpyrifos ethyl & $23,8 \mathrm{c}$ & $40,6 \mathrm{~d}$ & $31,7 \mathrm{c}$ & $37,2 \mathrm{c}$ & $9,8 \mathrm{a}$ & $4,68 \mathrm{c}$ & $1,56 \mathrm{c}$ \\
\hline 15. foram + iodo + novarulon & $20,0 \mathrm{~d}$ & $4,2 \mathrm{~g}$ & $46,3 \mathrm{a}$ & $40,6 \mathrm{a}$ & $9,4 \mathrm{a}$ & $8,01 \mathrm{~b}$ & $3,36 \mathrm{a}$ \\
\hline 16. mesotrione ${ }^{74}$ & $5,6 \mathrm{~d}$ & $3,0 \mathrm{~g}$ & $47,5 \mathrm{a}$ & $39,6 \mathrm{~b}$ & $9,6 \mathrm{a}$ & $10,17 \mathrm{a}$ & $3,19 \mathrm{~b}$ \\
\hline 17. mesotrione + permethrin & $4,6 \mathrm{e}$ & $3,0 \mathrm{~g}$ & $48,5 \mathrm{a}$ & $39,4 \mathrm{~b}$ & $10,0 \mathrm{a}$ & $10,23 \mathrm{a}$ & $3,81 \mathrm{a}$ \\
\hline 18. mesotrione + methomyl & $4,4 \mathrm{e}$ & $3,0 \mathrm{~g}$ & $49,2 \mathrm{a}$ & $40,2 \mathrm{a}$ & $10,0 \mathrm{a}$ & $10,07 \mathrm{a}$ & $3,43 \mathrm{a}$ \\
\hline 19. mesotrione + chlorpyrifos ethyl & $48,0 \mathrm{~b}$ & $21,0 \mathrm{f}$ & $39,9 \mathrm{~b}$ & $35,4 \mathrm{~d}$ & $9,0 \mathrm{~b}$ & $4,10 \mathrm{~d}$ & $1,48 \mathrm{c}$ \\
\hline 20. mesotrione + novarulon & $6,8 \mathrm{e}$ & $3,2 \mathrm{~g}$ & $48,1 \mathrm{a}$ & $38,0 \mathrm{c}$ & $9,8 \mathrm{a}$ & $9,60 \mathrm{a}$ & $3,62 \mathrm{a}$ \\
\hline 21. carfentrazone ${ }^{15}$ & $21,0 \mathrm{~d}$ & $29,4 \mathrm{e}$ & $38,5 \mathrm{~b}$ & $36,0 \mathrm{~d}$ & $9,8 \mathrm{a}$ & $3,35 \mathrm{~d}$ & $2,37 \mathrm{~b}$ \\
\hline 22. carfentrazone + permethrin & $24,0 \mathrm{c}$ & $34,0 \mathrm{e}$ & $41,7 \mathrm{a}$ & $33,2 \mathrm{e}$ & $9,0 \mathrm{~b}$ & $3,48 \mathrm{~d}$ & $1,19 \mathrm{c}$ \\
\hline 23. carfentrazone + methomyl & $22,0 \mathrm{~d}$ & $26,6 \mathrm{e}$ & $38,6 \mathrm{~b}$ & $36,0 \mathrm{~d}$ & $9,2 \mathrm{~b}$ & $4,81 \mathrm{c}$ & $1,50 \mathrm{c}$ \\
\hline 24. carfentrazone + chlorpyrifos ethyl & $78,0 \mathrm{a}$ & $79,0 \mathrm{a}$ & $24,6 \mathrm{~d}$ & $15,6 \mathrm{f}$ & $6,8 \mathrm{c}$ & $1,39 \mathrm{e}$ & $0,48 \mathrm{~d}$ \\
\hline 25. carfentrazone + novarulon & $22,6 \mathrm{~d}$ & $28,2 \mathrm{e}$ & $35,5 \mathrm{~b}$ & $36,8 \mathrm{c}$ & $9,4 \mathrm{a}$ & $4,01 \mathrm{~d}$ & $1,25 \mathrm{c}$ \\
\hline $26.2,4-D^{-16}$ & $26,6 \mathrm{c}$ & $40,0 \mathrm{~d}$ & $46,7 \mathrm{a}$ & $41,0 \mathrm{a}$ & $8,8 \mathrm{~b}$ & $6,18 \mathrm{c}$ & $1,26 \mathrm{c}$ \\
\hline 27. 2,4-D + permethrin & $25,0 \mathrm{c}$ & $52,0 \mathrm{c}$ & $47,4 \mathrm{a}$ & $41,6 \mathrm{a}$ & $8,8 \mathrm{~b}$ & $5,77 \mathrm{c}$ & $1,18 \mathrm{c}$ \\
\hline 28. 2,4-D + methomyl & $24,0 \mathrm{c}$ & $42,0 \mathrm{~d}$ & 47,9 a & $41,8 \mathrm{a}$ & $8,8 \mathrm{~b}$ & $5,27 \mathrm{c}$ & $1,27 \mathrm{c}$ \\
\hline 29. 2,4-D + chlorpyrifos ethyl & $20,0 \mathrm{~d}$ & $63,0 \mathrm{~b}$ & $48,7 \mathrm{a}$ & $40,4 \mathrm{a}$ & $8,6 \mathrm{~b}$ & $5,12 \mathrm{c}$ & $1,12 \mathrm{c}$ \\
\hline 30. 2,4-D + novarulon & $26,8 \mathrm{c}$ & $41,6 \mathrm{~d}$ & $46,9 \mathrm{a}$ & $41,8 \mathrm{a}$ & $8,6 \mathrm{~b}$ & $5,41 \mathrm{c}$ & $1,74 \mathrm{c}$ \\
\hline 31. testemunha sem aplicação & $0,0 \mathrm{f}$ & $0,0 \mathrm{~g}$ & $48,6 \mathrm{a}$ & $41,6 \mathrm{a}$ & $10,0 \mathrm{a}$ & 11,86 a & $3,64 \mathrm{a}$ \\
\hline F & $1351,0 *$ & $2472,8^{*}$ & $199,0^{*}$ & $111,0^{*}$ & $2,37 *$ & $40,66^{*}$ & $4,92 *$ \\
\hline C.V. $(\%)$ & 23,29 & 29,46 & 9,97 & 3,61 & 5,86 & 16,25 & 21,72 \\
\hline
\end{tabular}

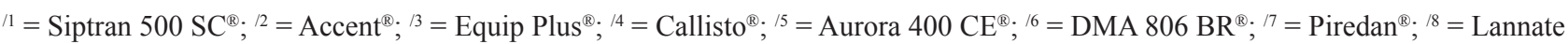
$\mathrm{BR}^{\circledR} ;{ }^{19}=$ Lorsban $480 \mathrm{BR}^{\circledR} ; / 10=$ Gallaxy $100 \mathrm{EC}^{\circledR}$. Médias seguidas de mesma letra na coluna não diferem entre si pelo teste de agrupamento de Scott-Knott, em nível de 5\% de probabilidade. $(*=$ significativo; ns = não significativo) 
semelhantemente ao ocorrido no estádio $\mathrm{V}_{3}$, manteve-se como o tratamento mais prejudicial para todas as variáveis estudadas.

Em geral, os resultados demonstraram que a mistura do inseticida chlorpyrifos ethyl (organofosforado) não apresenta efeito deletério às plantas de milho apenas quando associados aos herbicidas inibidores de ALS. O inseticida chlorpyrifos ethyl também afetou negativamente a seletividade inicial quando associado ao carfentrazone, mesotrione e 2,4-D, independentemente do híbrido ou estádio fenológico das plantas no momento da aplicação. Portanto, em função das misturas em tanque de herbicidas e inseticidas serem comuns nos mais diferentes níveis de tecnológicos de produção de milho, faz-se necessário o desenvolvimento de novos estudos sobre essa temática, assim com a disponibilização das informações para que seu uso não envolva riscos desnecessários.

Por fim, destaca-se que a condução de experimentos em condição de campo é fundamental para certificação dos resultados, já que os efeitos prejudiciais observados inicialmente ao crescimento do milho podem não refletir redução significativa de produtividade ao final do ciclo da cultura. Nesse contexto, é importante ressaltar que para seletividade de herbicidas só é possível estabelecer conclusões adequadas com a avaliação da produtividade, já que existe a necessidade de avaliar se os efeitos dos sintomas visualmente detectáveis e de outros imperceptíveis sobre o crescimento realmente afetam a produtividade da cultura.

\section{Conclusões}

As misturas em tanque entre os herbicidas e inseticidas atrazine + permethrin e nicosulfuron + novarulon não prejudicaram o crescimento inicial do milho híbrido $30 \mathrm{~F} 35$ para aplicação no estádio $\mathrm{V}_{3}$, ao contrário de nicosulfuron, mesotrione e 2,4-D associados ao chlorpyrifos ethyl, as quais reduziram significativamente a maioria das variáveis analisadas.

Todas as misturas em tanque realizadas no estádio $\mathrm{V}_{6}$ reduziram significativamente ao menos uma variável relacionada ao crescimento inicial do híbrido 30F35.

Os tratamentos com atrazine e suas misturas com inseticidas não prejudicaram o crescimento inicial do híbrido 2B-710 na aplicação realizada em $\mathrm{V}_{3}$, ao contrário do ocorrido no estádio $\mathrm{V}_{6}$, onde apenas a aplicação isolada de atrazine e da mistura de mesotrione + metomyl não causaram redução significativas das variáveis analisadas.

A mistura entre cafentrazone + chlorpyrifos ethyl foi extremamente prejudicial ao crescimento inicial do híbrido 2B-710, tanto na aplicação no estágio $\mathrm{V}_{3}$ quanto em $\mathrm{V}_{6}$.

\section{Referências}

AZANIA, C. A. M.; AZANIA, A. A. P. M. Seletividade de herbicidas. In: MONQUERO, P. A. (Ed.). Plantas daninhas: aspectos da biologia e manejo de plantas daninhas. São Carlos: RiMa, 2014. p. 217-233.

CARVALHO, S. J. P.; NICOLAI, M.; FERREIRA, R. R.; FIGUEIRA, A. V. O.; CHRISTOFFOLETI, P. J. Herbicide selectivity by differential metabolism: considerations for reducing crop damages. Scientia Agricola, Piracicaba, v. 66, n. 1, p. 136-142, 2009.

DOI: 10.1590/S0103-90162009000100020.

CAVALIERI, S. D.; SILVA, F. M. L.; VELINI, E. D.; SÃO JOSÉ, A. R.; ULLOA, S. M.; DATTA, A.; CAVALIERI, J. D.; KNEZEVIC, S. Z. Seletividade do nicosulfuron em três estádios fenológicos do milho pipoca. Planta Daninha, Viçosa, MG, v. 30, n. 2, p. 377-386, 2012.

DOI: $10.1590 / \mathrm{S} 0100-83582012000200017$. 
CHERIFI, M.; RAVETON, M.; PICCIOCCHI, A.; RAVANEL, P.; TISSUT, M. Atrazine metabolism in corn seedlings. Plant Physiology and Biochemistry, Paris, v. 39, n. 7/8, p. 664-672, 2001.

DOI: $10.1016 /$ S0981-9428(01)01281-5.

DALAZEN, G.; MARKUS, C.; KASPARY, T. E.; PISONI, A.; GALLON, M.; QUEIROZ, A. R. S.; VIDAL, R. A.; MEROTTO JÚNIOR, A. Occurrence and importance of herbicide resistance caused by degradation enhancement for weed management. Revista Brasileira de Herbicidas, Londrina, v. 15, n. 1, p. 26-38, 2016.

DIEHL, K. E.; STOLLER, E. W.; BARRETT, M. In vivo and in vitro inhibition of nicosulfuron metabolism by terbufos metabolites in maize. Pesticide Biochemistry and Physiology, San Diego, v. 51, n. 2, p. 137-149, 1995. DOI: $10.1006 /$ pest.1995.1014.

GAZZIERO, D. L. P. Misturas de agrotóxicos em tanque nas propriedades agrícolas do Brasil. Planta Daninha, Viçosa, MG, v. 33, n. 1, p. 83-92, 2015.

DOI: $10.1590 / \mathrm{S} 0100-83582015000100010$.

KARAM, D.; OLIVEIRA, M. F. de. Seletividade de herbicidas na cultura do milho. Sete Lagoas: Embrapa Milho e Sorgo, 2007. 8 p. (Embrapa Milho e Sorgo. Circular Técnica, 98).

KOEPPE, M. K.; HIRATA, C. M.; BROWN, H. M.; KENYON, W. H.; O'KEEFE, D. P.; LAU, S. C.; ZIMMERMAN, W. T.; GREEN, J. M. Basis of selectivity of the herbicide rimsulfuron in maize. Pesticide Biochemistry and Physiology, San Diego, v. 66, n. 3, p. 170-181, 2000. DOI: 10.1006/pest.1999.2470.

KREUZ, K.; FONN-PFISTER, R. Herbicide-insecticide interaction in maize: malathion inhibits cytochrome P450-dependent primisulfuron metabolism. Pesticide Biochemistry and Physiology, San Diego, v. 43, n. 3, p. 232-240, 1992. DOI: 10.1016/0048-3575(92)90036-Y.

LÓPEZ-OVEJERO, R. F.; FANCELLI, A. L.; DOURADO NETO, D.; GARCÍA Y GARCÍA, A.; CHRISTOFFOLETI, P. J. Seletividade de herbicidas para cultura de milho (Zea mays) aplicados em diferentes estádios fenológicos da cultura. Planta Daninha, Viçosa, MG, v. 21, n. 3, p. 413419, 2003. DOI: 10.1590/S0100-83582003000300009.

MATZENBACHER, F. O.; BORTOLY, E. D.; KALSING, A.; MEROTTO, A. Distribution and analysis of the mechanisms of resistance of barnyardgrass (Echinochloa crus-galli) to imidazolinone and quinclorac herbicides. The Journal of Agricultural Science, Cambridge, v. 153, n. 6, p. 1044-1058, 2015.

DOI: $10.1017 / \mathrm{S} 0021859614000768$.

NELSON, D.; WERCK-REICHHART, D. A P450 - centric view of plant evolution. The Plant Journal, Oxford, v. 66, n. 1, p. 194-211, 2011.

DOI: 10.1111/j.1365-313X.2011.04529.x.

NICOLAI, M.; CARVALHO, S. J. P.; LÓPEZ-OVEJERO, R. F.; CHRISTOFFOLETI, P. J. Aplicação conjunta de herbicida e inseticida na cultura do milho. Bragantia, Campinas, v. 65, n. 3, p. 413-420, 2006.

DOI: $10.1590 / \mathrm{S} 0006-87052006000300007$.

OGLIARI, J.; FREITAS, S. P.; EVARISTO, J. A. E.; GOMES, S. A.; OKOROKOVA FAÇANHA, A. L.; RETAMAL, C. A.; FAÇANHA, A. R. Análise do perfil eletroforético de proteínas citoplasmáticas para verificação do processo de desintoxicação do herbicida mesotrione em plantas de Zea mays. Planta Daninha, Viçosa, MG, v. 32, n. 1, p. 161-172, 2014.

DOI: $10.1590 / \mathrm{S} 0100-83582014000100018$.

RAVETON, M.; RAVANEL, P.; SERRE, A. M.; NURIT, F.; TISSUT, M. Kinetcs of uptake and metabolism of atrazine in model plant systems. Pesticide Science, Oxford, v. 49, n. 2 , p. $157-163,1997$.

DOI: 10.1002/(SICI)1096-9063(199702)49:2<157::AID-PS517>3.0.CO;2-M.

REZENDE, B. P. M.; TAVARES, C. J.; MARANGONI, R. E.; CUNHA, P. C. R.; JAKELAITIS, A. Efeito do fomesafen + fluazifop-p-butil associados com inseticidas no controle das plantas daninhas na cultura da soja. Revista Brasileira de Ciências Agrárias, Recife, v. 7, n. 4, p. 608613, 2012.

DOI: 10.5039/agraria.v7i4a1842. 
SILVA, A. A.; FREITAS, F. M.; FERREIRA, L. R.; JAKELAITIS, A.; SILVA, A. F. Aplicações sequenciais e épocas de aplicações de herbicidas em misturas com chlorpirifos no milho e em plantas daninhas. Planta Daninha, Viçosa, MG, v. 23, n. 3, p. 527-534, 2005. DOI: $10.1590 / \mathrm{S} 0100-83582005000300017$.

SILVA, A. A.; JAKELAITIS, A.; FREITAS, F. M.; FERREIRA, L. R.; VIVIAN, R. Interação entre herbicida e inseticida sobre o milho-pipoca (Zea mays), as plantas daninhas e a lagarta-do-cartucho. Planta Daninha, Viçosa, MG, v. 25, n. 1, p. 181-186, 2007.

DOI: $10.1590 / \mathrm{S} 0100-83582007000100020$.

SPADER, V.; VIDAL, R. A. Seletividade e dose de injúria econômica de nicosulfuron aplicados em diferentes estádios de desenvolvimento da cultura do milho. Ciência Rural, Santa Maria, v. 31, n. 6, p. 929-934, 2001. DOI: 10.1590/S0103-84782001000600001.

STECKEL, L. E.; STEWART, S. D.; STECKEL, S. Corn response to post-applied HPPD-inhibitor based premix herbicides with in-furrow and foliar-applied insecticide. Weed Technology, Alabama, v. 29, n. 1, p. 18-23, 2015. DOI: 10.1614/WT-D-14-00030.1

TREZZI, M. M.; FELIPPI, C. L.; NUNES, A. L.; CARNIELETO, C. E.; FERREIRA, A. R. J. Eficácia de controle de plantas daninhas e toxicidade ao milho da mistura de foramsulfuron e iodosulfuron isoladamente ou em associação com atrazine e/ou clorpirifós. Planta Daninha, Viçosa, MG, v. 23, n. 4, p. 653-659, 2005. DOI: $10.1590 / \mathrm{S} 0100-83582005000400013$.
VELINI, E. D.; OSIPE, R.; GAZZIERO, D. L. P. (Coord.). Procedimentos para instalação, avaliação e análise de experimentos com herbicidas. Londrina: SBCP, 1995.42p.

VIDAL, R. A.; MEROTTO JÚNIOR, A.; SCHAEDLER, C. E.; LAMEGO, F. P.; PORTUGAL, J.; MENENDES, J.; KOZLOWSKI, L. A.; TREZZI, M. M.; PRADO, R. Mecanismo de ação dos herbicidas. In: MONQUERO, P. A. (Ed.). Plantas daninhas: aspectos da biologia e manejo de plantas daninhas. São Carlos: RiMa, 2014. p. 235-256.

VIEIRA JÚNIOR, N. S.; JAKELAITIS, A.; CARDOSO, I. S. Associação de herbicidas aplicados em pós-emergência na cultura do milho. Global Science and Technology, Goiânia, v. 8, n. 1, p. 1-8, 2015.

WERCK-REICHHART, D.; HEHN, A.; DIDIERJEAN, L. Cytochromes P450 for engineering herbicide tolerance. Trends in Plant Science, Cambridge, v. 5, n. 3, p. 116123, 2000. DOI: 10.1016/S1360-1385(00)01567-3.

YASOUR, H.; OSUNA, M. D.; ORTIZ, A.; SALDAÍN, N. E.; ECKERT, J. W.; FISCHER, A. J. Mechanism of resistance to penoxsulam in late watergrass [Echinochloa phyllopogon (Stapf) Koss.]. Journal of Agricultural and Food Chemistry, Easton, v. 57, n. 9, p. 3653-3660, 2009. DOI: $10.1021 / \mathrm{jf} 8039999$.

ZANATTA, J. F.; MANFREDI-COIMBRA, S.; PROCÓPIO, S. O.; MANICA-BERTO, R.; SGANZERLA, D. C.; CARNEIRO, J. C. Interações entre herbicidas e inseticidas na cultura do algodão: uma revisão. Revista da FZVA, Uruguaiana, v. 14, n. 2, p. 34-45, 2007. 